\title{
Achieving Equity in Telehealth: "Centering at the Margins" in Access, Provision, and Reimbursement
}

\author{
Andrea Westby, MD, Tanner Nissly, DO, Rebecca Gieseker, MD, \\ Kaleigh Timmins, MD, and Kathryn Justesen, MD
}

The SARS-CoV-2 epidemic has led to rapid transformation of health care delivery and access with increased provision of telehealth services despite previously identified barriers and limitations to this care. While telehealth was initially envisioned to increase equitable access to care for under-resourced populations, the way in which telehealth provision is designed and implemented may result in worsening disparities if not thoughtfully done. This commentary seeks to demonstrate the opportunities for telehealth equity based on past research, recent developments, and a recent patient experience case example highlighting benefits of telehealth care in underserved patient populations. Recommendations to improve equity in telehealth provision include improved virtual visit technology with a focus on patient ease of use, strategies to increase access to video visit equipment, universal broadband wireless, and inclusion of telephone visits in CMS reimbursement criteria for telehealth. ( $\mathrm{J}$ Am Board Fam Med 2021;34:S29-S32.)

Keywords: Access to Health Care, Centers for Medicare and Medicaid Services, CovID-19, Medically Underserved Area, Telemedicine

Telehealth, defined by the World Health Organization $(\mathrm{WHO})$ as the provision of health care services where patients and providers are separated by distance, ${ }^{1}$ has held the promise of equity in access to care since its implementation in the United States in the 1970s. Opportunities to reach communities and populations that lacked access to high quality health care seemed limitless. However, in the following decades, increasing insurance and governmental regulations led to telehealth being designed, marketed, and implemented for communities and populations already well-resourced. This then led to enhancing the

This article was externally peer reviewed.

Submitted 8 June 2020; revised 24 July 2020; accepted 3 August 2020.

From North Memorial Family Medicine Residency/ Broadway Family Medicine, Department of Family Medicine and Community Health, University of Minnesota Medical School, Minneapolis, MN (AW, TN, KJ); PGY-2, North Memorial Family Medicine Residency/Broadway Family Medicine, Department of Family Medicine and Community Health, University of Minnesota Medical School, Minneapolis, MN (RG, KT).

Funding: There was no funding for this project.

Conflicts of interest: We have no conflicting or competing interests.

Corresponding author: Andrea Westby, MD, 2426 W Broadway Ave, Minneapolis, MN 55411 (Email: westby@ umn.edu). health of the majority and already healthier populations through the offering of telemedicine concierge services, private partnerships, and "expanded access for those who can pay for expanded access." ${ }^{2}$ In addition, the Center for Medicare and Medicaid Services (CMS) defines telehealth more narrowly than $\mathrm{WHO}^{3}$ only including visits with real time face-toface interaction, that is, video, and excluding telephone visits, potentially limiting the reimbursement of certain types of visits and disproportionately impacting under-resourced populations.

In response to the SARS-CoV-2 epidemic, ambulatory medicine practices have rapidly implemented telehealth options to continue caring for populations while limiting risk of exposure to the virus for health care providers and patients. Preexisting telehealth guidelines and reimbursements which had previously limited the breadth of impact in many settings have rapidly shifted in response. However, policymakers currently state that these changes are temporary to provide relief for the duration of the pandemic. ${ }^{4}$ Based on our clinical experiences to date, we feel telehealth offers ongoing opportunity to improve equity in health care access beyond the pandemic provided a few key improvements are made. 
When the public health crisis of SARS-CoV-2 became an immediate threat to the health and safety of providers, staff, and patients, the University of Minnesota Broadway Family Medicine Clinic in Minneapolis promptly transitioned the majority of our in-person office visits to telephone or video visits, regardless of the potential for reimbursement. Within the first 8 weeks of offering phone and video visits, providers at our clinic performed 2766 patient visits. Of these visits, 1692 were by phone, 498 were by video, and 576 were in-person. Medical students, family medicine residents, and our community health worker identified patients over the age of 60 with high-risk health conditions such as diabetes, chronic obstructive pulmonary disease, and heart disease and conducted outreach by telephone to discuss the virus and services currently being offered at Broadway. This outreach led to a sense of safety and community among patients and providers, and as a result, many patients who had not been seen in the clinic in over a year scheduled and completed telephone visits. Here is 1 case example of a patient served by telehealth:

$D M$ is a 61-year-old male with type 2 diabetes and cardiovascular disease with a bistory of limited clinic follow up. He was last seen in clinic in Fanuary of 2019 after hospitalization for acute pancreatitis and uncontrolled diabetes. DM has limited access to transportation, making traveling to the clinic a challenge. As part of our outreach to bigh-risk patients during the SARS-CoV-2 epidemic, our clinic's community bealth worker contacted bim and scheduled him for a telephone visit, as he had concerns about not being able to use the technology for a video visit. DM also did not read well, a barrier to care that he had not disclosed to anyone in the clinic previously and was not documented in his chart. Because be was in his own home and speaking to a provider on the phone, DM felt comfortable disclosing his challenges with reading and was able to spell the medication names directly from his medication bottles to the provider. He was not aware that some of his medications were supposed to be taken twice a day (including shortacting metformin and carvedilol) because be could not read the instructions on the bottle. Because he had his medication bottles in front of him while at home, DM was able to directly mark the twice-daily medications, which increases the likelihood that he will adhere to the prescribed treatment regimen. He was also able to come in for a lab visit at his convenience and was glad to schedule a follow-up telephone visit in 2 weeks to review his medication changes.
As seen in the case of DM, telephone communication is accessible, efficient, and well-accepted by patients in our underserved clinic setting. In addition, providing telephone visits has increased access to primary care that was limited in our patient population by barriers such as those faced by DM. Studies on telehealth barriers have assumed that certain "individual" level factors are barriers that cannot be overcome by clinical or systemic innovation. However, in 2019 Antonio et al discussed the importance of not misinterpreting demographic factors as individual, unavoidable predictors of health outcomes. Instead, they recommend viewing these demographic factors as "social determinants of health inequities," to emphasize that they are socially mediated rather than personal or individual. ${ }^{5}$

Underserved populations often have known barriers to face-to-face telehealth visits: lack of access to video equipment, lack of reliable internet, concern about cost of copays, concern about cost of wireless data consumption, unfamiliarity with virtual platforms, confidentiality, and trust concerns, and language barriers. ${ }^{6}$ Despite these obstacles, we have seen the implementation of telehealth at our clinic as an opportunity to increase provision of high-quality primary care to underserved and under-resourced communities. Transportation issues, insufficient childcare, and limited ability to take time off work to attend an in-office visit have been mitigated by telehealth options. Other barriers, including transit time, time spent in the clinic, and mental health barriers such as agoraphobia or depression have been significantly decreased if not completely eliminated by telehealth. As our clinic has limited visitors attending in-person office visits, telehealth provides the opportunity for family members and caregivers to contribute to the history and decision making if the patient desires this additional support.

To provide true equity of care, we must center at the margins: we must make the system work for the people and communities who experience inequities, both before and now exacerbated by the pandemic. "Centering at the margins," a central tenet of critical race theory, refers to "making the perspectives of socially marginalized groups, rather than those of people belonging to dominant race or culture, the central axis around which discourse on a topic revolves."7

If telehealth is accessible for those who are presumed the least able to use it, it will also work for those who do not have similar limitations. We need 
to ask our patients and communities what limits their access to telehealth primary care, both video and telephone, and address the limitations through systemic changes to progress toward equitable access to health care.

From our evaluation of the literature and the information gained during the rapid implementation of telemedicine during the SARS-CoV-2 pandemic in early 2020, we propose the following considerations to increase the likelihood of equitable telehealth care:

1. Healthcare providers need to utilize telehealth platforms that are easy to use even for the most technology-challenged patients and do not incur additional costs or barriers to use.

2. Payers should reimburse telephone visits at the same rate as face-to-face video and in-person visits to ensure equity of care provision. People who cannot access video visits should not be relegated to in-person office visits as their only option at this time, as this could be a barrier to quality primary care. Additionally, to remain financially solvent, health care systems need assurance that adequate reimbursements for telehealth will persist beyond the pandemic and include higher reimbursement for initial video visits to account for time spent orienting the patient to the technology being used.

3. Video visits must be an accessible option for all patients who desire them. Many significant structural barriers must be overcome for this to be a reality, including access to highspeed broadband internet, video-capable devices, and an appropriate environment from which to have a visit. Potential options for providing the necessary resources for video visits include:

a. Dispense video-capable devices or webcams by insurance companies, clinics/healthcare providers, or governmental agencies with whom patients come into contact.

b. Add video hubs staffed by community health workers (CHWs) in high rises, homeless shelters, or community centers. From 1972 to 1975, Mt. Sinai Hospital established and operated an experimental telemedicine-enabled community health site in a public assistance housing project in East Harlem, which was ultimately terminated due to lack of funding. ${ }^{2}$

c. Provide high-speed broadband internet access for all people. Consider free wireless internet options in parking lots of grocery stores, post offices, big box stores, etc. One example already in place is in Washington state, where the state encouraged grocery stores and Wal-Marts to expand their free wireless internet access and make it more user-friendly. ${ }^{8}$ Additionally, companies such as Comcast have adjusted their business plans to ensure internet access through the current pandemic. ${ }^{9}$

4. Remember that some of the barriers to video visits are individual level and not easily overcome by healthcare system action, policy change, or systemic procedures, and thus telephone visits must be an option for these patients.

Currently, there is no documented research showing a difference in quality between in-person, telephone, and video visits in primary care. Further studies will need to be done to examine outcomes in more detail, as until the SARS-CoV-2 pandemic, telehealth primary care, particularly telephone primary care, was not provided widely. This investigation must include how the lack of physical examination affects quality of care, and if we can trust provider knowledge and expertise to guide which conditions warrant a physical examination to provide appropriate care. We would argue that if done thoughtfully, telephone visits will increase access to primary care without compromising quality. Minimizing barriers to telehealth for all communities and populations will provide increased access to health care, thereby leading us closer to health equity for all.

To see this article online, please go to: http://jabfm.org/content/ 34/Supplement/S29.full.

\section{References}

1. World Health Organization. Telehealth. Available at https:/www.who.int/gho/goe/telehealth/en/. Published November 8, 2019. Accessed June 2, 2020.

2. Greene JA. As telemedicine surges, will community health suffer? Boston Rev. Available at http://bostonre view.net/science-nature/jeremy-greene-telemedicinesurges-will-community-health-suffer. Published April13, 2020. Accessed June 2, 2020.

3. Centers of Medicare and Medicaid Services.Medicare telehealth health care provider fact sheet. Available at https://www.cms.gov/newsroom/fact-sheets/medicaretelemedicine-health-care-provider-fact-sheet. Published March 17, 2020. Accessed June 2, 2020. 
4. US Health and Human Services, Public Affairs. Telehealth: delivering care safely during COVID-19. Available at https://www.hhs.gov/coronavirus/telehealth/ index.html\#billing. Published May 7, 2020. Accessed June 2, 2020.

5. Antonio MG, Petrovskaya O. Towards Developing an eHealth Equity Conceptual Framework. Stud Health Technol Inform 2019;257:24-30.

6. Scott Kruse C, Karem P, Shifflett K, Vegi L, Ravi K, Brooks M. Evaluating barriers to adopting telemedicine worldwide: A systematic review. J Telemed Telecare 2018;24:4-12.

7. Ford CL, Airhihenbuwa CO. Critical race theory, race equity, and public health: Toward antiracism praxis. Am J Public Health 2010;100:S30-5.

8. Klein S, Hostetter M. Rapid tech deployment to reach vulnerable residents: A Q\&A with the Washington State
Health Care Authority. Milbank Memorial Fund. Available at https:/www.milbank.org/news/rapid-techdeployment-to-reach-vulnerable-residents-a-qawith-washington-states-health-care-authority/?utm_ medium=email\&utm_campaign=The Milbank Monthly April 2020\&utm_content=The Milbank Monthly April 2020 CID_5220edab3c7902ad192980c0f70479c0\&utm source=Email Campaign Monitor\&utm_term=Rapid Tech Deployment to Reach Vulnerable Residents A QA with the Washington State Health Care Authority. Published April 2, 2020. Accessed June 3, 2020.

9. Comcast. Comcast announces comprehensive COVID19 response to help keep Americans connected to the internet. MassTLC. Available at https://www. masstlc.org/comcast-announces-comprehensive-covid19-response-to-help-keep-americans-connected-to-theinternet/. Published March 19, 2020. Accessed June 3, 2020 . 